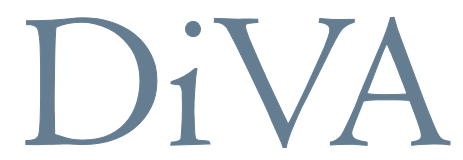

http://uu.diva-portal.org

This is a submitted version of a paper published in Optics Communications.

Citation for the published paper:

Tuncer, Enis \& Niklasson, Gunnar A.

"Optical properties of non-dilute metal-insulator composites"

Optics Communications, 2008, Vol. 281, Issue 17: 4374-4379

URL: http://dx.doi.org/10.1016/j.optcom.2008.05.020

Access to the published version may require subscription.

Published with permission from: Elsevier 


\title{
Optical properties of non-dilute metal-insulator composites
}

\author{
Enis Tuncer* \\ Applied Superconductivity Group, Fusion Energy Division, Oak Ridge National \\ Laboratory, Oak Ridge, TN 37831-6122, USA
}

Gunnar A. Niklasson

Department of Engineering Sciences, The Solid State Physics, The Angström Laboratory, Uppsala University, SE 75121 Uppsala Sweden

\begin{abstract}
The description of the optical properties of metal-insulator composites in the non-dilute region is a long standing problem. In this letter we extract the spectral density function of cobalt-amorphous aluminum oxide composites from optical and near-infrared data. The spectral functions are accurately computed numerically with the help of a recently developed technique. It is observed that the spectral features of the prepared composites change with increasing cobalt content. For low concentrations of cobalt, only one depolarization peak is found that corresponds to the Maxwell Garnett approximation. For concentrations higher than $11 \%$ cobalt, three effective depolarization factors are resolved that move towards low spectral parameter values with increasing cobalt content. Such a multi-peak structure arises naturally in fractal equivalent circuit models for the optical properties. A comparison with a deterministic fractal model is presented to illustrate the strength of the spectral density representation and to better comprehend our results. We conclude that the observed behavior gives important information on the relation of the optical characteristics to the composite micro-structure.
\end{abstract}

Key words: Spectral density representation, dielectric permittivity, composite, optical properties, fractals

PACS: 77.22.-d, 78.20.-e, 77.22.Ch, 77.84.Lf, 02.70.Hm, 05.45.Df

\footnotetext{
* Author to whom any correspondence should be addressed. Sponsored by the U.S. Department of Energy - Office of Electricity Delivery and Energy Reliability, Superconductivity Program for Electric Power Systems under contract DE-AC05-00OR22725 with Oak Ridge National Laboratory, managed and operated by UT-Battelle, LLC.

Email addresses: tuncere@ornl.gov (Enis Tuncer), gunnar.niklasson@angstrom. uu. se (Gunnar A. Niklasson).
} 


\section{Introduction}

For many decades the structure-property relationship in composite materials has been sought by researchers in various fields, e.g., physics, materials science, geophysics, pharmaceutics, biology, etc., in order to comprehend the nature of material mixtures. Such a deep understanding would be of great value for calculating either the dielectric constant of a mixture of substances of known dielectric permittivities or, knowing the dielectric permittivities of a mixture of two components and that of one of the components, to calculate the dielectric permittivity of the other [1], or even knowing the dielectric permittivities of a mixture and those of the components to estimate the morphology of the mixture $[2,3]$. Consequently concepts like fractal geometry and morphology of dense mixtures can be studied by investigating their frequency dependent electrical and optical properties. In metal-insulator composites, the optical properties can be varied from those of the metal to those of the insulator, by simply changing the composition. In the dilute metal or insulator limit the optical properties are understood in detail and can be described by effective medium theories [4-7]. However, there exist a more or less broad transition region [7] at intermediate concentrations where the structure is interconnected and ill-defined. At the percolation threshold (metal-insulator transition) the universal scaling behavior is well-understood [13], and also been experimentally observed [8]. However, for composites with particle sizes of a few $\mathrm{nm}$ and at ambient temperatures, the percolation transition is frequently very smeared out and difficult to observe [8]. It appears that further studies are necessary in order to arrive a consistant description of the optical and electrical properties of metal-insulator composites in the transition region.

In principle an exact description of the optical properties of non-dilute composites would require an exact knowledge of the nano-structure-something that can not be realized in practice. However, the scaling at the percolation threshold is not predicted to be sensitively dependent on the local structural arrangements. In practice, the effects of the nano-structure are difficult to observe directly because they are obscured by the frequency dependence of the dielectric permittivities of the constituents. The spectral density formalism [9] gives a rigorous descripition of the dielectric permittivity of two-component composites, where the influence of the nano-structure is given in terms of a spectral density function (SDF). All possible effective medium theories based on microstructure are special cases of this formalism, each represented by its own SDF. Hence, by this formalism it is possible to separate the effects of the nano-structure from the optical properties of the constituents. It is thus also possible to study directly the structural resonances of the composite provided that the SDF can be obtained from the experimental data.

In this Letter we use a novel highly efficient recently developed numerical method

[2] to extract the spectral density function of cobalt-amorphous aluminum oxide $\left(\mathrm{Co}-\mathrm{Al}_{2} \mathrm{O}_{3}\right)$ composites from the experimental data [6] on the dielectric permit- 
tivity in the optical and near-infrared wavelength ranges. The applied numerical method is based on the Bayesian statistics approach, in which spectral parameters $(x)$ are selected randomly, and the SDF estimates are computed many times, using the Monte Carlo integration hypothesis, with a new set of $x$-values in each step (for details see Refs. [2] and citeTuncer2000b). Other methods based on regularization techniques have also been developed to solve similar problems [11]. Specifically we apply Fuchs spectral density representation [9] (based on a distribution of depolarization factors of small particles) for the topological, structural description, of Co- $\mathrm{Al}_{2} \mathrm{O}_{3}[6]$. We show that the spectral density in the percolation region exhibits a multi-peak structure. Such a structure can be interpreted in terms of fractal equivalent circuit models for the optical properties [12-14]. Our novel results open a way to explicitly connect features of the spectral density to structure-based models for the optical transition region.

\section{Computational method}

We now present the spectral density representation for a two-component material. It is implicitly assumed that the optical and electrical properties of the composite are due solely to a nano-structure dependent averaging of the properties of the constituents. In the spectral density representation, the dielectric response $\varepsilon_{\mathrm{e}}$ of a heterogeneous material is written in the complex 'scaled' permittivity $\xi$ form as follows [2, 3]

$$
\begin{aligned}
\xi & =\left(\varepsilon_{\mathrm{e}}-\varepsilon_{\mathrm{m}}\right)\left(\varepsilon_{\mathrm{i}}-\varepsilon_{\mathrm{m}}\right)^{-1} \\
& =\xi_{s}+\int_{0^{+}}^{1} \mathrm{~g}(x)\left[1+\left(\varepsilon_{\mathrm{i}} \varepsilon_{\mathrm{m}}^{-1}-1\right) x\right]^{-1} \mathrm{~d} x
\end{aligned}
$$

Here, subscripts $\mathrm{e}, \mathrm{m}$ and $\mathrm{i}$ denote the effective, matrix and inclusion media, $x$ is the spectral parameter, $\mathrm{g}(x)$ is the $\mathrm{SDF}$ (which is analogous to the distribution of depolarization factors of Fuchs [9]), and $\xi_{s}$ is the percolation strength, which contains the contribution of $\mathrm{g}(x \equiv 0)$. The mathematical properties of $\mathrm{g}$ are expressed by the following sum rules; $\xi_{s}+\int_{0^{+}}^{1} \mathrm{~g}(x) \mathrm{d} x=q$ and $\int_{0^{+}}^{1} x \mathrm{~g}(x) \mathrm{d} x=(1-q) d^{-1}$, where $d$ is the dimensionality of the system related to the effective depolarization factor $\eta$ of inclusions, $\eta=d^{-1}$, and $q$ is the fractional Co content. Once the SDF is known, the effective depolarization factors $\eta$, giving a morphological description, of the metal phase can be estimated by using the two sum rules. The first summation rule yields the concentation of the specific shape distribution, and the second summation rule indicates the most expected depolarization factor, or namely the shape of the metalic inclusions. Therefore, the two sum rules describe the system geometry, which contains specific information on the metallic inclusions shape and contribution, or the volume fraction of particular shape. For example, in a mixture with well-defined mono dispersed inclusions, the SDF yields a single peak [2]. However, experimen- 
tal data on brine-porous rock mixture had several peaks in its SDF which were correlated to the structure of porous in the rock. Later in the test, it is shown that the resolved parameters for different peaks in a Co- $\mathrm{Al}_{2} \mathrm{O}_{3}$ SDF can be inserted in a mixture formula to simulate the complex dielectric function of the non-dilute metalinsulator mixture. Both the numerical method and the mixture formula have previously been applied to a logarithmic mixing law (in arXiv: cond-mat 0503750 [2]) that has shown the utility of the procedure. The first summation rule has been explicitly illustrated that the sum of $\xi_{s}$ and $\int \mathrm{g}(x) \mathrm{d} x(\equiv\langle q\rangle)$ has yielded the actual concentration of the conducting phase for both approaches. Note that in the current study no percolation strength has been estimated for the $\mathrm{Co}-\mathrm{Al}_{2} \mathrm{O}_{3}$ from the experimental optical data.

It should be noted that the effective depolarization factors are not simply related to particle shape, but also describe the detailed multipolar interactions between the cobalt particles. Some examples of depolarization factor estimates for two dimensional structures and for a brine-porous rock mixture [2] have recently been reported using the impedance data from ab initio computer simulations and from literature[17]. In the computations 11 different lattice arrangements with unidirectional fibers have been considered. The SDF resolved have illustrated the importance of lattice arrangement and multipolar interactions in these systems. The SDF resolved from the experimental data of the brine-porous rock mixture revealed shape and concentration of pores in the rock, which is an important factor to risk analyze oil recovery in an oil reserve.

In the present investigations, the spectral parameter space is chosen to be in logarithmic scale. The pre-selected random $x$ values are taken from a log-linear distribution $-3 \leq \log _{10} x \leq 0$ (see Ref. [10] for details), then the percolation strength $\xi_{s}=\int_{-\infty}^{-3} \mathrm{~g}\left(\log _{10} x\right) \mathrm{d} \log _{10} x$. The extracted SDFs are in the form of single or sum of individual distributions depending on the volume fraction of cobalt in the composite, for this reason we analyze the resulting peak(s) by employing the Lévy statistics [15],

$$
\mathrm{L}\left(\log _{10} x ; \mathfrak{A}, \mu, \mathfrak{g}, \mathfrak{z}\right)=\mathfrak{A}\left|\exp \left\{-\left|\mathfrak{z}\left(\log _{10} x-\mu\right)\right|^{\mathfrak{g}}\right\}\right|
$$

where, $\mathfrak{g}$ is the characteristic exponent; $\mu$ is the localization parameter; $\mathfrak{z}$ is the scale parameter, and $\mathfrak{A}$ is the amplitude. Lévy statistics is used for interacting systems in different fields (see Tuncer et al. ). The Lévy stable distribution is a natural generalization (approximation) of the normal (Gaussian), Cauchy or Lorenz and Gamma distributions. It is used when analyzing sums of independent identically distributed random variables by a diverging variance. The special forms of Eq. (3) are the Gaussian $\mathrm{L}\left(\log _{10} x ; \mathfrak{A}, \mu, 2, \mathfrak{z}\right)$, the Lorentz or Cauchy $\mathrm{L}\left(\log _{10} x ; \mathfrak{A}, \mu, 1, \mathfrak{z}\right)$ and Gamma $\mathrm{L}\left(\log _{10} x ; \mathfrak{A}, \mu, 1 / 2, \mathfrak{z}\right)$ distributions. This equation could be used to characterize the properties of the obtained SDF with $\mathfrak{A}$ corresponding to the amplitute of the mean depolarization strength. 
The measured dielectric permittivities of cobalt [18] and aluminum oxide [19] are used as $\varepsilon_{\mathrm{i}}$ and $\varepsilon_{\mathrm{m}}$ in Eq. (1) to estimate the SDFs of $\mathrm{Co}-\mathrm{Al}_{2} \mathrm{O}_{3}$ composite films with various Co contents. For very low concentration of cobalt in the mixture, the influence of the crystal field spectrum of cobalt, which is significant in the dielectric dispersion of the films [20], result in unsatisfactory SDF estimates. Therefore, we use a narrower wavelength $\lambda$ window and disregard the data for $\lambda<0.6 \mu \mathrm{m}$ [20] for composites with $q \leq 0.11$. For higher cobalt volume fractions, the whole wavelength window between $350 \mathrm{~nm}$ to $3000 \mathrm{~nm}$ is used in the numerical analysis. To extract the SDFs, the minimization (number of the Monte Carlo steps $N_{\mathrm{MC}}$ ) is performed $2^{13}$ times, later the results are binned in 256 channels between $-3 \leq \log x \leq 0$. The number of unknowns are selected as $95 \%$ of the number of experimental data points, $M$. A calculation time for a given volume fraction of cobalt is approx. $3 \mathrm{~min}$ on a Linux installed $1.5 \mathrm{GHz}$ Laptop computer with 512 Mbyte memory.

\section{Experiments}

In this section we give a brief review of the experimental characterization of our samples, which has been reported in detail before [6]. Thin films (thickness 30$80 \mathrm{~nm}$ ) of $\mathrm{Co}-\mathrm{Al}_{2} \mathrm{O}_{3}$ were deposited by electron-beam co-evaporation from separate cobalt and alumina sources. Film thicknesses and volume fractions were determined by an alpha-step surface profilometer from steps between areas of the sample, that were shaded from one or both of the deposition fluxes, as reported before [6]. Particle sizes of Co were determined by electron microscopy and were found to be in the range 1.5 to $3.5 \mathrm{~nm}$, increasing slightly with cobalt volume fraction, $\mathrm{q}$. Electron micrographs [6] show evidence for some short-range clustering, as found by analysis of pair distribution functions [22]. This feature was seen for the whole studied range of $\mathrm{q}$, but should be interpreted with caution since the micrographs show the projection of the particles in a $30 \mathrm{~nm}$ slice onto a plane. The electrical conductivity was measured in a planar configuration between two evaporated metal strips $[6,22]$. The temperature coefficient of resistance was negative for most volume fractions, crossing over to a positive value indicating metallic conduction at $\mathrm{q}=0.7[6]$.

The dielectric permittivity of films with q less than 0.73 was obtained from numerical inversion of near-normal optical transmittance and reflectance measurements, performed by a Beckman ACTA MVII spectrophotometer in the wavelength range 350 to $3000 \mathrm{~nm}$. Details of the analysis as well as permittivity spectra as a function of wavelength have been reported before [6]. For transition metal composites interband transitions in the metal smear out the structural resonances of the composite in the visible and near infrared ranges. In this case it is necessary to obtain the SDF from the permittivity data, in order to be able to characterize the influence of the composite nanostructure on the optical properties. 


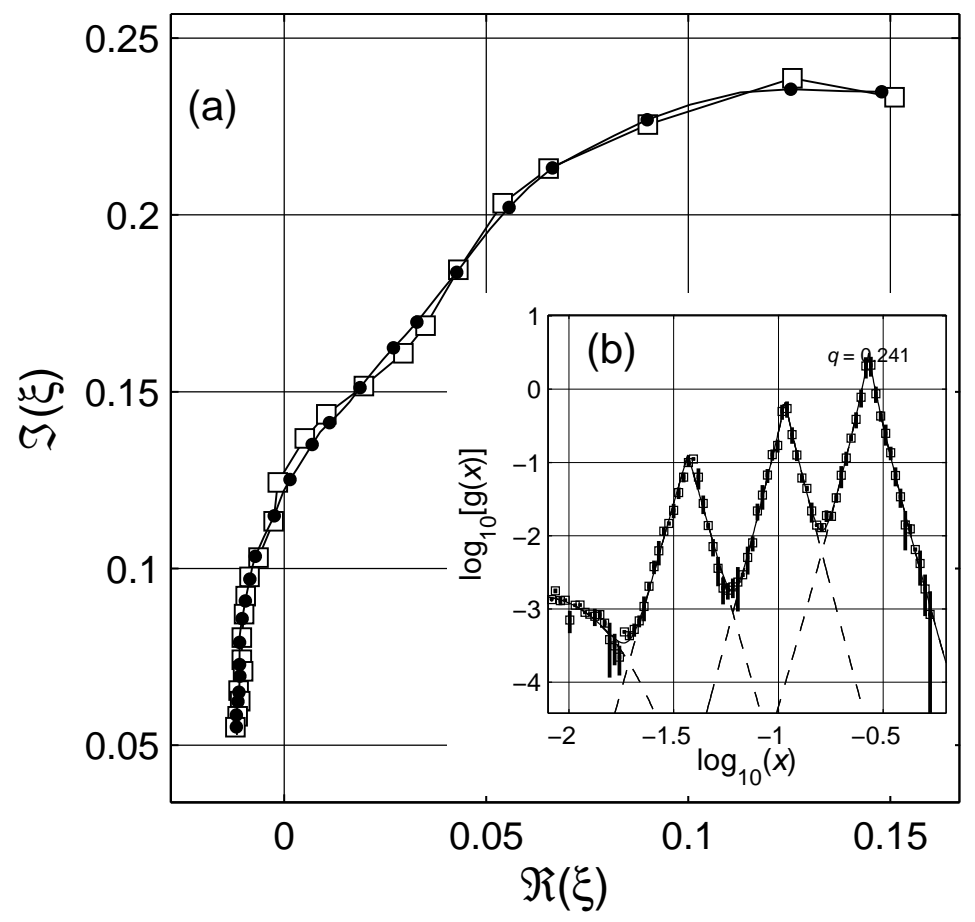

Fig. 1. (a) The Argand plot of the complex 'scaled' permittivity $\xi$ for $37 \%$ cobalt-doped aluminum oxide. The open symbols $(\square)$ are the measurements, and the filled points $(\bullet)$ are the $\xi$ estimates of the numerical analysis. The solid lines are drawn to guide the eye. (b) The extracted SDF for $37 \%$ cobalt-doped amorphous aluminum oxide. The estimates are presented with error bars and open symbols $(\square)$. The dashed lines $(---)$ shows the individual Lévy distributions for the resolved sub-density functions, and the solid line ( - ) is the sum of all sub-density functions.

\section{Results}

To illustrate the analysis steps and their significance, we explicitly give the results for the sample with $37 \%$ cobalt. In Fig. 1a, the Argand plot of the 'scaled' permittivity $\xi$ is shown. The extracted SDF has four distinct distributions (cf. Fig. 1b), which as mentioned before are analyzed with Lévy statistics. In the figure, the dashed lines $(---)$ represent the four Lévy distributions. Three of the distributions are sharp with $\mathfrak{g}$ close to one, resembling a Lorenz distribution (delta-sequence), the forth one at low $x$ values is very wide. Co concentration estimates for each of these spectral peaks are $0.001,0.011,0.047$ and 0.182 for peaks one to four numbered from low $x$ to high $x$ values (cf. Fig. 1b). The total concentration $\langle q\rangle$ of Co estimated from the spectral function $\left(\langle q\rangle=\int \mathrm{g}(x) \mathrm{d} x \approx 0.241\right)$ is lower than that determined experimentally, $q=0.37$ [6]. The deviation between these two Co concentration values will be discussed below.

The SDFs of composite films with different Co contents are presented in Fig. 2. They are shifted in the y-direction for clarity. In addition, the list of parameter estimates for the statistical analysis of the peak shapes is given in Table 1. For low 
volume fractions of Co, $q \leq 0.11$, the SDF exhibits just one peak, with an effective depolarization factor of 0.3 to 0.4 . The data for $q=0.03$ are more uncertain due to the strong influence of crystal field absorption [20]. In this region the SDF indicates a structure of isolated nearly spherical particles, and the Maxwell-Garnett approximation [4] gives a fairly good description of the optical properties [6]. However the large width of the SDF peak indicates significant effects of particle-particle interactions. In an intermediate range, $q=0.19-0.26$, the largest depolarization factor

Table 1

Parameters of the Lévy statistics and estimated topological information for cobalt-doped amorphous aluminum oxide cerments. The column ' $i$ ' shows the peak number for the spectral density function for the particular concentration level, as shown in Fig. 2. Quantities between angle paranthesis ' \langle\rangle ' are the expected values estimated from the statistical analysis, namely the average $x$, volume fraction and depolarization factor estimated for each peak. The percolation strengths $\xi_{s}$ calculated from measured dc conductivities are given in the last column.

\begin{tabular}{rrrrrr|rrr|r}
\hline \hline$q$ & $i$ & $\mathfrak{A}$ & $\mu$ & $\mathfrak{g}$ & $\mathfrak{z}$ & $\langle x\rangle$ & $\langle q\rangle$ & $\eta$ & $\xi_{s}$ \\
\hline 0.03 & 1 & 0.20 & -0.27 & 1.15 & 16.17 & 0.54 & 0.02 & 0.55 & \\
\hline 0.07 & 1 & 0.99 & -0.47 & 0.83 & 33.17 & 0.34 & 0.07 & 0.37 & \\
\hline 0.12 & 1 & 0.46 & -0.51 & 1.23 & 10.26 & 0.31 & 0.08 & 0.33 & \\
\hline 0.19 & 1 & 0.09 & -1.16 & 1.23 & 23.46 & 0.07 & 0.01 & 0.07 & \\
& 2 & 0.26 & -0.88 & 1.11 & 25.81 & 0.13 & 0.02 & 0.14 & \\
& 3 & 2.58 & -0.46 & 0.92 & 34.52 & 0.35 & 0.16 & 0.41 & \\
\hline 0.25 & 1 & 0.09 & -1.31 & 0.85 & 27.13 & 0.05 & 0.01 & 0.05 & \\
& 2 & 0.09 & -1.06 & 1.47 & 16.63 & 0.09 & 0.01 & 0.09 & \\
& 3 & 1.37 & -0.66 & 1.03 & 28.36 & 0.22 & 0.10 & 0.24 & \\
& 4 & 2.17 & -0.30 & 0.74 & 41.21 & 0.50 & 0.12 & 0.57 & \\
\hline 0.26 & 1 & 0.10 & -1.31 & 0.81 & 30.58 & 0.05 & 0.01 & 0.05 & \\
& 2 & 0.10 & -1.06 & 1.39 & 17.51 & 0.09 & 0.01 & 0.09 & \\
& 3 & 1.41 & -0.66 & 1.01 & 29.37 & 0.22 & 0.10 & 0.24 & \\
& 4 & 2.12 & -0.30 & 0.79 & 38.20 & 0.50 & 0.12 & 0.57 & \\
\hline 0.37 & 1 & 0.00 & -2.22 & 2.44 & 2.69 & 0.01 & 0.00 & 0.01 & 0.0005 \\
& 2 & 0.12 & -1.43 & 1.03 & 22.04 & 0.04 & 0.01 & 0.04 & \\
& 3 & 0.72 & -0.97 & 0.92 & 31.25 & 0.11 & 0.05 & 0.11 & \\
& 4 & 3.14 & -0.57 & 0.87 & 36.92 & 0.27 & 0.18 & 0.33 & \\
\hline 0.52 & 1 & 0.81 & -1.62 & 0.78 & 51.41 & 0.02 & 0.04 & 0.02 & 0.007 \\
& 2 & 0.91 & -1.03 & 0.95 & 30.39 & 0.09 & 0.06 & 0.10 & \\
& 3 & 2.92 & -0.64 & 0.95 & 31.51 & 0.23 & 0.19 & 0.28 & \\
\hline 0.56 & 1 & 0.38 & -1.71 & 0.82 & 39.87 & 0.02 & 0.02 & 0.02 & 0.02 \\
& 2 & 0.65 & -1.12 & 0.97 & 31.23 & 0.08 & 0.04 & 0.08 & \\
& 3 & 3.26 & -0.73 & 0.90 & 35.55 & 0.19 & 0.19 & 0.23 & \\
\hline 0.57 & 1 & 0.25 & -1.71 & 1.03 & 24.55 & 0.02 & 0.02 & 0.02 & \\
& 2 & 0.52 & -1.12 & 1.10 & 25.15 & 0.08 & 0.04 & 0.08 & \\
& 3 & 3.08 & -0.73 & 0.93 & 33.18 & 0.19 & 0.19 & 0.23 & \\
\hline 0.71 & 1 & 1.32 & -1.84 & 1.06 & 25.61 & 0.01 & 0.10 & 0.02 & 0.32 \\
& 2 & 0.21 & -1.28 & 1.20 & 23.53 & 0.05 & 0.02 & 0.05 & \\
& 3 & 3.96 & -0.85 & 0.91 & 36.10 & 0.14 & 0.23 & 0.18 & \\
\hline & & & & & & & & & \\
\end{tabular}




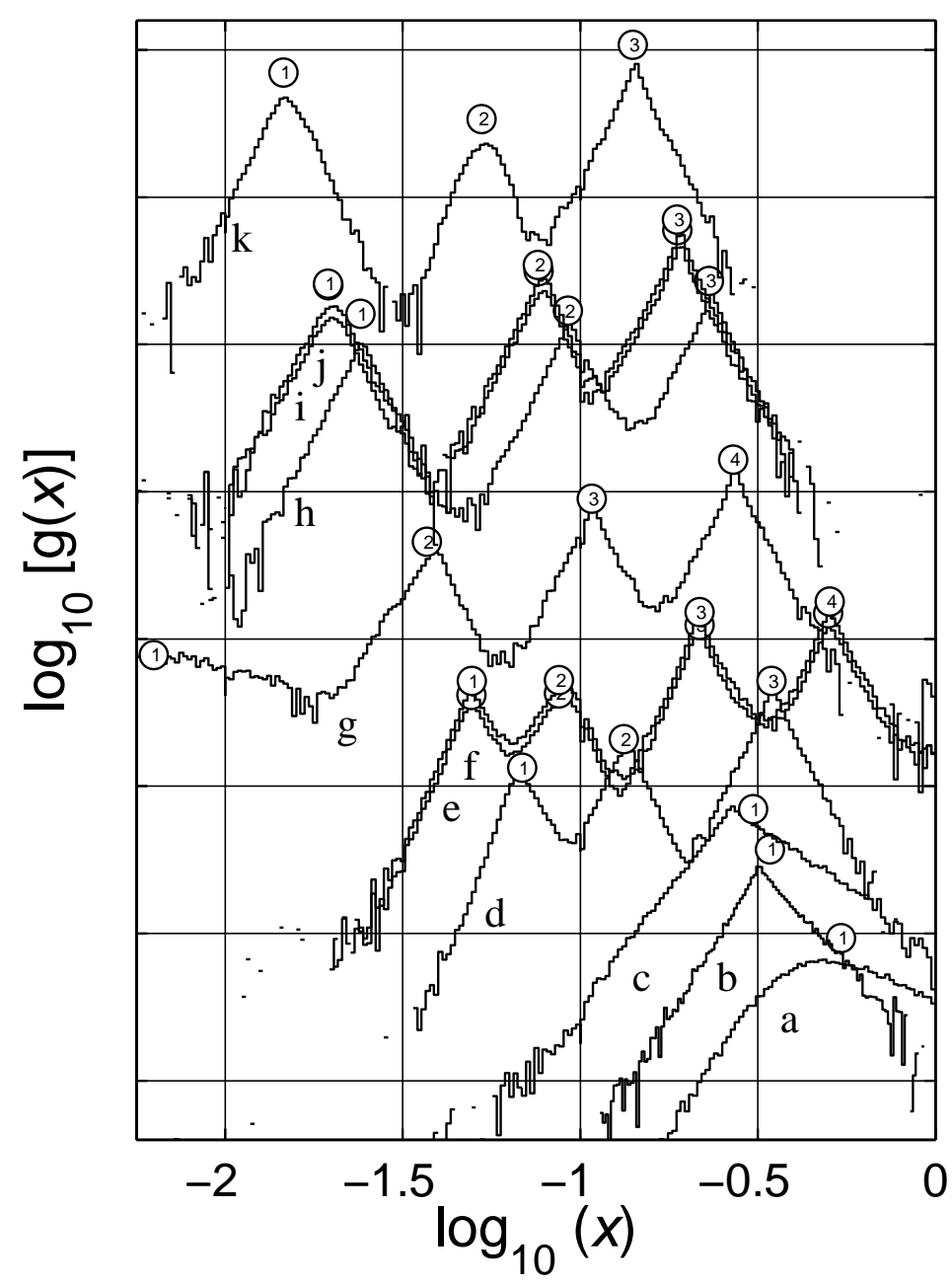

Fig. 2. The extracted SDFs for cobalt-doped amorphous aluminum oxide. The data are shifted vertically depending on the concentration of cobalt for clarity. The letters denote the Co concentration; a:0.03, b:0.07, c:0.12, d:0.19, e:0.25, f:0.26, g:0.37, h:0.52, i:0.56, $\mathrm{j}: 0.57, \mathrm{k}: 0.71$. Observe that the number of peaks increase for cermets with higher volume fractions of cobalt than 0.11 and similar Co concentrations yield the same spectra. The peaks are numbered from left to right.

is shifted to higher values, which is probably due to anisotropy due to formation of clusters of particles. In addition a multi-peak structure develops in the SDF, indicating the clustering of particles to form larger structures. The estimated $q$ corresponds well with the experimentally measured one for all samples with $q<0.3$.

In Fig. 3, the depolarization factors are illustrated as a function of Co concentration. The development of the individual depolarization peaks could be characterized as located in three branches, labeled with $A, B$ and $C$ in Fig. 3. A major change in the depolarization structure of the material can clearly be seen at $0.17<q \leq 0.37$.

For samples with still higher volume fractions, we have entered what we denote the optical transition region [21]. There are now three peaks in the SDF, all posi- 


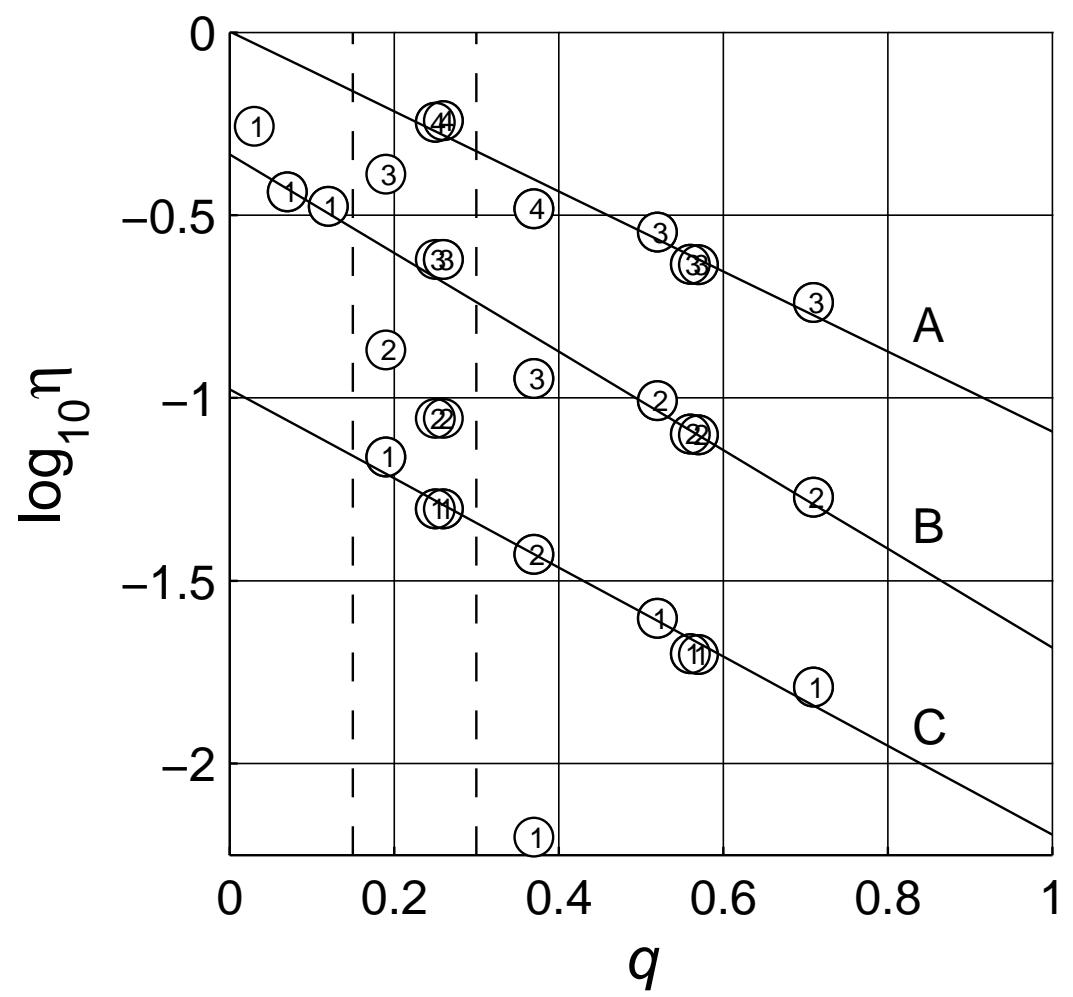

Fig. 3. The estimated depolarition factors $\eta\left[=\langle x\rangle(1-q)^{-1}\right]$ from individual peaks of the statistical analysis. The two vertical dashed lines $(---)$ separate the particular regions that the spectral density function indicate optical transition region. The log-linear lines are drawn to guide the eyes, the slopes of $A, B$ and $C$ are $-1.02,-1.31$ and -1.22 , respectively.

tioned at depolarization factors lower than that of a non-interacting sphere. This clearly rules out an interpretation in terms of single particle shape. The peaks in the SDF gradually shift to lower $\eta$ as the volume fraction of Co increases. Interestingly, the ratio between adjacent peak positions is nearly constant for the samples with $q \geq 0.37$. These qualitative features of the SDF show a similarity to the behavior of fractal equivalent circuit models of optical properties of percolating composites $[13,14]$. These models display a series of resonances in the optical absorption and the ratio of the frequencies of adjacent resonances is approximately constant $[13,14]$. We suggest that such conceptual models capture the essentials of the structure of our $\mathrm{Co}-\mathrm{Al}_{2} \mathrm{O}_{3}$ composites in this optical transition region. Particles cluster together more and more as $q$ increases, leading to a shift of the effective depolarization factors to lower values. However, the conductivity is still not metallic [21], which means that the percolating clusters are quite small and separated by tunneling barriers or thin layers of the insulator phase. We suggest that such geometry can be described by the first stages of the fractal network construction. It has been shown before that fractal network resonances are very difficult to observe in optical absorption data [23]. However, the SDF representing the experimental data in terms of the SDF allows us to determine the structural resonances and thus avoids these limitations. 


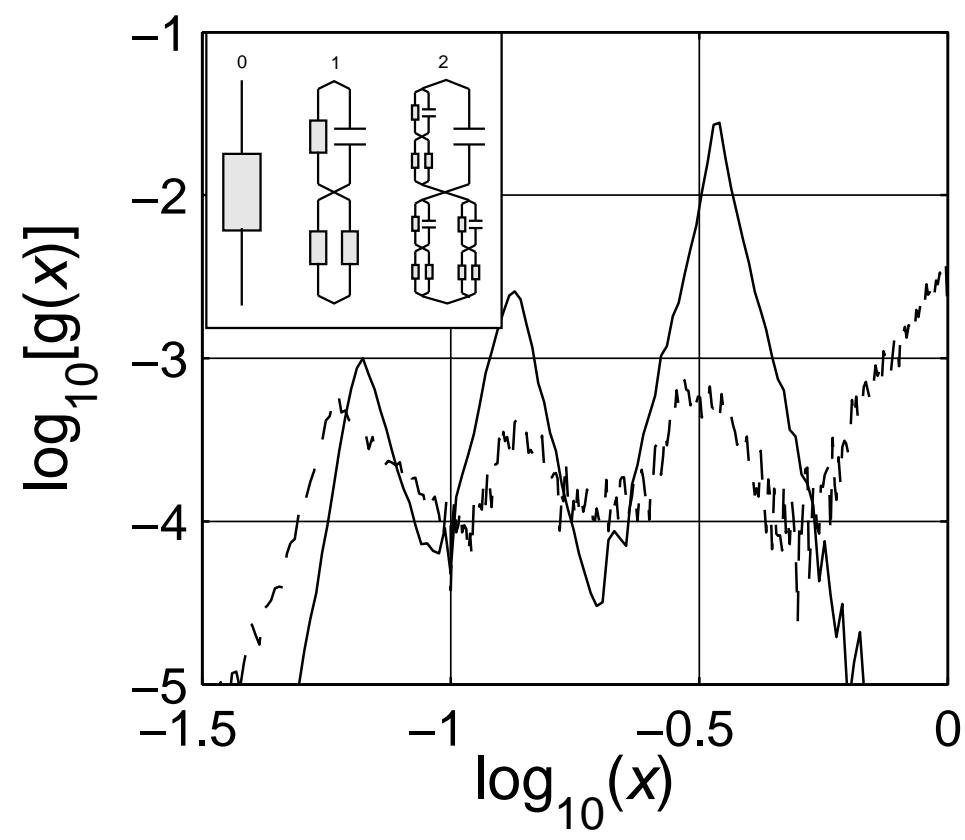

Fig. 4. Comparison of deconvoluted SDFs obtained from experimental data ( - ) and the deterministic fractal impedance (DFIN) model $(---)$. The inset illustrates the first two stages of the construction of the DFIN. At stage $n$ it consists of three impedances of the (n-1)-th stage, each weighted by a factor $f / 3$ and a capacitance weighted with a factor $(1-f)$. In the computation we use $n=4$ and $f=(2 / 3)$, which gives a volume fraction of metal $q=f^{n} \sim 0.197$, approximately the same as the experimental one.

In the inset of Fig.4 we display a deterministic fractal impedance network (DFIN) model similar to that presented by Clerc et al.[13]. The first stage of the construction is built up from impedances ('conducting (Co)' bonds) and capacitances ('dielectric $\left(\mathrm{Al}_{2} \mathrm{O}_{3}\right)$ ' bonds). The construction is then iterated so that at the $n$-th stage, it consists of three impedances of the $n-1$-th stage and a capacitance (see inset in Fig. 4a). The impedance of the network can easily be calculated recursively [13]. In Fig. 4 we compare the function $\mathrm{g}(x)$ obtained from the DFIN at stage $n=4$ using $q=(2 / 3)^{n} \equiv 0.197$ with the SDF obtained from the experimental data presented for the sample with $q=0.19$ in Fig. 2. It can be seen that the estimated SDF of the DFIN has several peaks, depending on the stage number, and that they are qualitatively similar to the experimental SDF of $\mathrm{Co}-\mathrm{Al}_{2} \mathrm{O}_{3}$. However, a fully qualitative aggreement can hardly be expected, since networks applicable to $\mathrm{Co}-\mathrm{Al}_{2} \mathrm{O}_{3}$ films will certainly be more complex than the simple cases treated in Refs. [12-14] and above. We emphasize that further work leading to extensions of these models is needed in order to obtain a quantitative description of the optical properties.

As mentioned above in the discussion of Fig. 1b, the volume fractions estimated from the SDF fall below the experimentally measured ones, when $q>0.3$. Fig. 5 shows that this behavior is consistently observed for the whole optical transition region. In theory, the deviation between the two curves should indicate the percolation strength $\xi_{s}$. The percolation strength can be estimated from the dc conductivity 


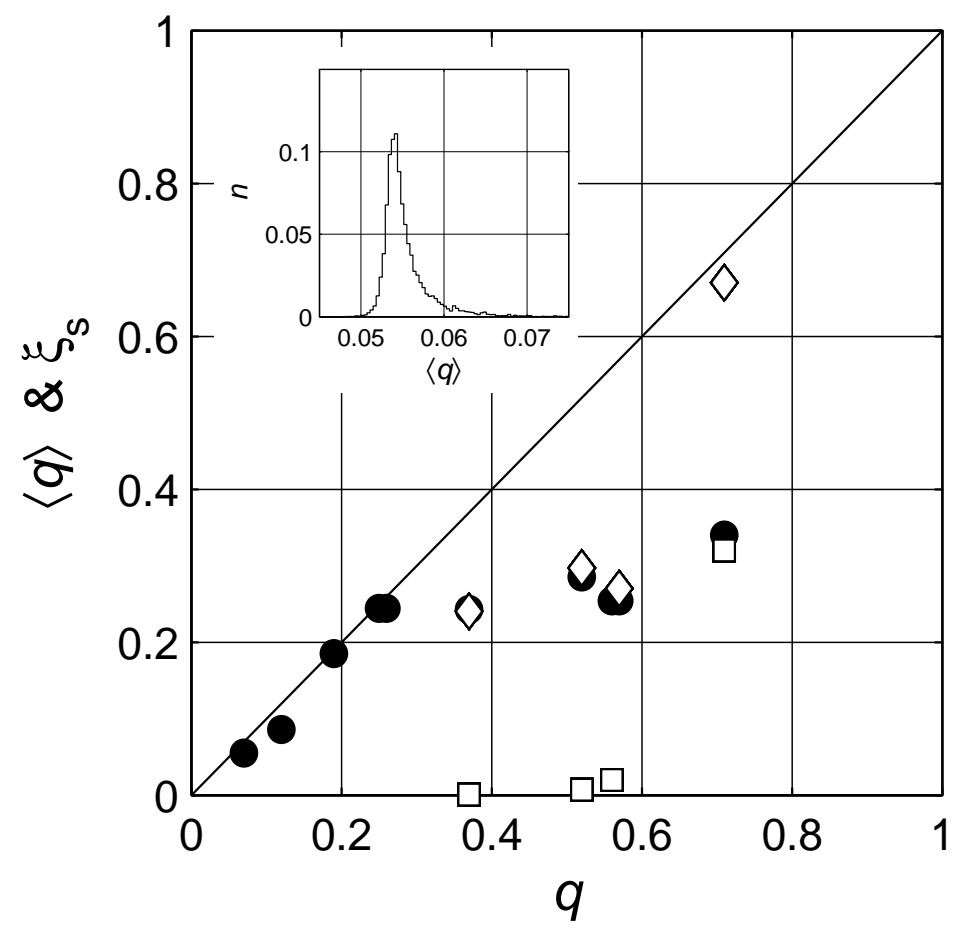

Fig. 5. The estimated volume fraction $\langle q\rangle$ of cobalt in the mixture versus the actual volume fraction $q$, represented with filled circles $(\bullet)$. The solid line $(-)$ indicates the $1: 1$ relation. The inset shows the number density distribution of the estimated cobalt volume fraction $\langle q\rangle$ in each Monte Carlo step, the actual concentration $q$ of cobalt is in this case 0.06. The open squares $(\square)$ are the percolation strengths estimated from the dc conductivity measurements. Observe that no $\xi_{s}$ is resolved from the SDF, analysis. The open lozenge $(\diamond)$ are the sume of the estimated volume fraction $\langle q\rangle$ and the percolation strengths $\xi_{s}$.

values for our films, previously given in Ref.[21]. It is given by the experimentally measured dc conductivity divided by the conductivity of the metallic phase, taking the grain size to be equal to the particle size. The percolation strength is very low for $0.2<q<0.6$, increasing steeply up to 0.02 at $q=0.6$, as given in Table 1 . Only for the films with $q=0.73$ do we obtain a percolation strength of 0.32 , which is at a level needed to bridge the gap in Fig. 5. This film displays evidence for metallic conduction [21], while the low frequency electrical properties of Co$\mathrm{Al}_{2} \mathrm{O}_{3}$ films with $0.3<q<0.6$ have been shown to be dominated by tunneling of electrons through thin barries between the metal particles [21]. Hence it seems that the percolation threshold of the $\mathrm{Co}-\mathrm{Al}_{2} \mathrm{O}_{3}$ films, which seems to occur in the region $0.6<q<0.7$ is coincident with the approach for the estimated $\langle q\rangle$ to the experiment one in Fig. 5. In the region where the electrical properties are dominated by tunneling and the temperature coefficient of resistance is negative, the dc conductivity can be modeled by a network consisting of metallic tunneling bonds between particles [24]. In this case the dc conductivity should be modeled by a network consisting of metallic and tunnelling bonds between the particles, as shown by Mantese et al.[24]. In the framework of the SDF representation, the composite thus consist of three components in this range of $q$, namely those associated with the metallic, 
insulator and tunneling contributions. Forcing a fit to a two-component SDF as in Eq. (1), will likely underestimate $\xi_{s}$ and $\langle q\rangle$, like in Fig. 5, since the SDF formalism implicitly assumes that the dc conductivity is due solely to the metal particles. The experimentally verified [21] tunneling behavior is not an average of metal and insulator electrical properties and is thus not described by the SDF formalism.

In order to illustrate and to strengthen the argument related to the present data analysis method and its significance we show that the complex dielectric function can be very well fitted to a simple mixture formula which takes into account the three major peaks observed in the SDF. The analysis is carried out on data Co$\mathrm{Al}_{2} \mathrm{O}_{3}$ films with a volume fraction in the region where $\langle q\rangle$ is underestimated in Fig. 5, namely for $37 \%$ Co. The mixture formula used is a compact representation (see Tuncer [25]) of Maxwell-Garnett formula and equal to a model derived from Wiener bounds [26]. In that case, assuming that the resolved $\mathrm{g}(x)$ are like Dirac delta functions, integration in Eq. (1) becomes a summation over number if peaks $i$,

$$
\xi \approx \sum_{i}\left\langle q_{i}\right\rangle\left[1+\left(\varepsilon_{\mathrm{i}} \varepsilon_{\mathrm{m}}^{-1}-1\right)\left\langle x_{i}\right\rangle\right]^{-1}
$$

One needs to make an adjustment for $\xi_{s}$ for the system since each depolarization generates a finite shift in real part of $\xi$ values, for details see [2]. Application of this procedure to complex dielectric permittivity of cermet with $37 \%$ Co is shown in Fig. 6. The individual depolarization curves are labeled as in Fig. 2 and Table 1.

\section{Conclusions}

In this Letter, we have used the spectral density representation to make thorough analysis of optical data for $\mathrm{Co}-\mathrm{Al}_{2} \mathrm{O}_{3}$ composite films, in a wide composition range. A recently developed numerical method resolved the spectral functions with unparalleled accuracy. The numerical method is a very convenient and accurate alternative to earlier methods. Features in the SDF that were not visible in the measured permittivity of $\mathrm{Co}-\mathrm{Al}_{2} \mathrm{O}_{3}$ could be resolved. In particular, we have resolved a series of structural resonances that were obscured by the dispersions of the permittivity of the constituents in the original data. Hence, our most important novel result is that for volume fractions $\geq 0.2$, the SDF displays a multi-peak structure. In a wide optical transition region, $0.3 \leq q \leq 0.7$ three peaks are clearly seen. We interpret these features as the behavior characteristic of fractal impedance network models, which show characteristic similarities with experimental data. We propose that this kind of model should be used to describe the optical properties of nearly percolating almost metallic composites. 

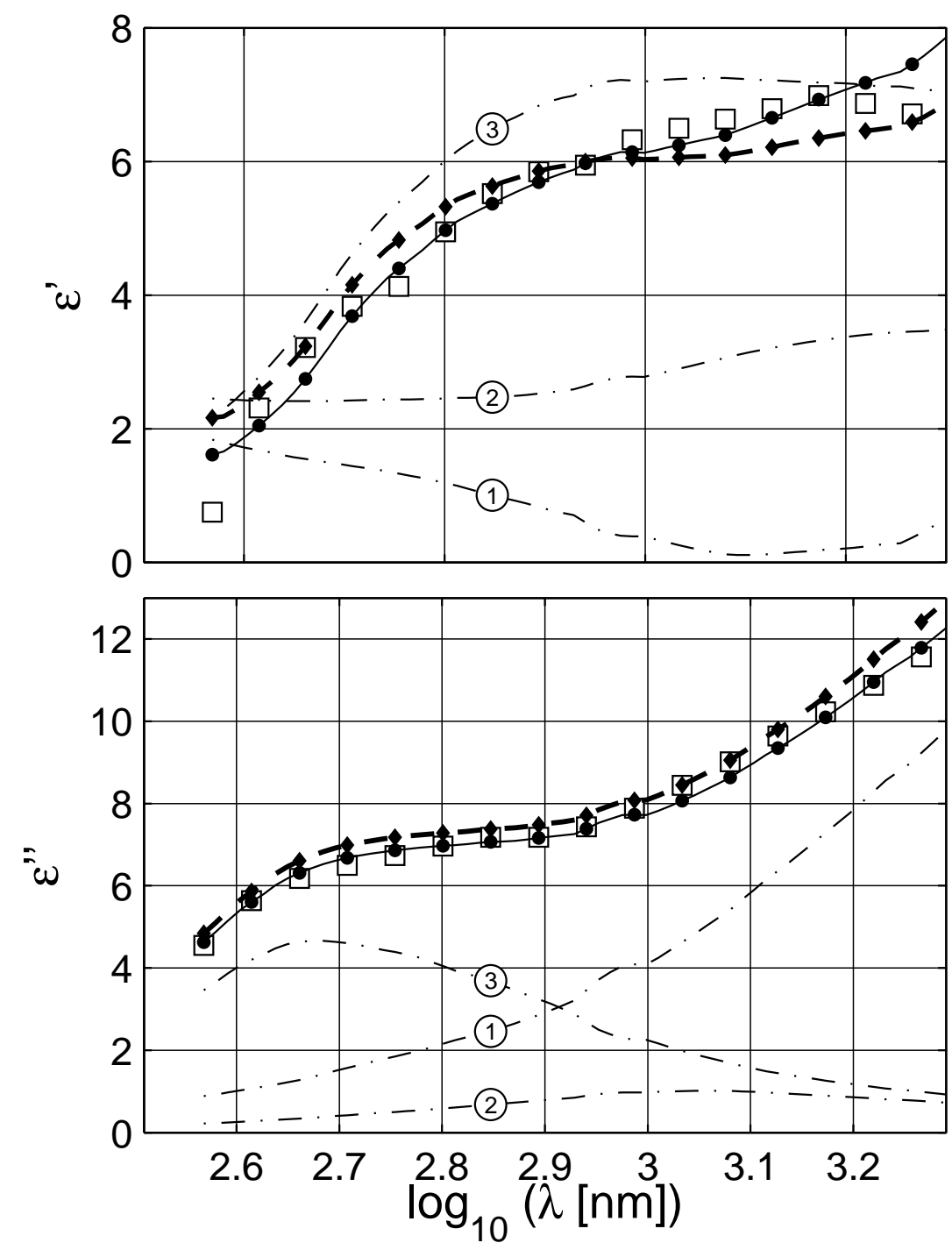

Fig. 6. (a) The real and (b) the imaginary parts of the dielectric permittivity of the sample with the highest volume fraction of cobalt. The measurements are plotted with open symbols $(\square)$. The permittivity estimated from the spectral density function after estimation of shift is the solid line (-) with filled circles $(\bullet)$. The sum of the three the depolarization expresions are shown with thick dashed line (- - ). The individual permittivities calculated with the expression in Eq. (4) are presented with chain lines $(-\cdot-)$, the numbers on the curves denote the peak numbers in Table 1.

\section{References}

[1] H. H. Lowry, J. Franklin Inst. 203, 413 (1927).

[2] E. Tuncer, Phys. Rev. B 71, 012101 (2005); E. Tuncer, J. Phys. D:Appl. Phys. 38, 223 (2005); E. Tuncer, arXiv: cond-mat/ 0503750.

[3] E. Tuncer, J. Phys.: Condens. Matter 17, L125 (2005); E. Tuncer, Phil. Mag. Lett. 85, 269 (2005).

[4] J. C. M. Garnett, Phil. Trans. Roy. Soc.London A 203, 385 (1904); O. Levy 
and D. Stroud, Phys. Rev. B 56, 8035 (1997).

[5] D. A. G. Bruggeman, Ann. Phys. (Leipzig) 24, 636 (1935).

[6] G. A. Niklasson and C. G. Granqvist, J. Appl. Phys. 55, 3382 (1984);

[7] B. Abeles, P. Sheng, M. D. Coutts, and Y. Arie, Adv. Phys. 24, 407 (1975).

[8] B. Abeles, H. L. Pinch, and J. I. Gittleman, Phys. Rev. Lett. 35(4), 247 (1975).

[9] R. Fuchs, Phys. Rev. B 11, 1732 (1975); D. J. Bergman, Phys. Rep. 43, 377 (1978); D. J. Bergman, Phys. Rev. Lett. 44, 1285 (1980); D. J. Bergman, Ann. Phys. 138, 78 (1982); G. W. Milton, J. Appl. Phys. 52, 5286 (1981); G. W. Milton, J. Appl. Phys. 52, 5294 (1981).

[10] E. Tuncer, Lic. thesis-Tech. rep. 338 L, Dept. of Electric Power Eng., Chalmers University of Technology, Gothenburg, Sweden (2000), ch. 5 p6383; E. Tuncer and S. M. Gubański, IEEE Trans. Dielect. Elect. Insul. 8, 310 (2001).

[11] A. R. Day, A. R. Grant, A. J. Sievers, and M. F. Thorpe, Phys. Rev. Lett. 84, 1978 (2000); A. R. Day and M. F. Thorpe, J. Phys.: Condens. Matter 11, 2551 (1999); E. Cherkaev and D. Zhang, Physica B 338, 16 (2003).

[12] J. A. Sotelo, V. N. Pustovit, and G. A. Niklasson, Phys. Rev. B 65, 245113 (2002).

[13] J. P. Clerc, G. Giraud, J. M. Laugier, and J. M. Luck, Adv. Phys. 39, 191 (1990); F. Brouers, D. Rauw, J. P. Clerc, and G. Giraud, Phys. Rev. B 49, 14582 (1994).

[14] P. M. Hui and D. Stroud, Phys. Rev. B 33, 2163 (1986). I. H. H. Zabel and D. Stroud, Phys. Rev. B 46, 8132 (1992).

[15] W. Feller, An Introduction to Probability Theory and Its Applications, vol. 2 (John Wiley and Sons, New York, 1970); L. Breiman, Probability, AddisonWesley Series in Statistics (Addison-Wesley Publishing Company, Inc., Reading, 1968). The special forms of Eq. (3) are the Gaussian $(\mathfrak{g}=2)$, the Lorentz or Cauchy $(\mathfrak{g}=1)$ and Gamma $\left(\mathfrak{g}=2^{-1}\right)$ distributions.

[16] E. Tuncer, M. Furlani, and B.-E. Mellander, J. Appl. Phys. 95(6), 3131 (2004).

[17] W. E. Kenyon, J. Appl. Phys. 55, 3153 (1984).

[18] G. A. Bolotin, M. M. Noskov, and I. I. Sasovskaya, Fiz. Metal. Metalloved 35, 699 (1973), [Phys. Metals Metallogr. 3524 (1973)]; P. B. Johnson and R. W. Christy, Phys. Rev. B 9, 5056 (1974).

[19] T. S. Eriksson, A. Hjortsberg, G. A. Niklasson, and C. G. Granqvist, Appl. Opt. 20, 2742 (1981).

[20] G. A. Niklasson, J. Appl. Phys. 57, 157 (1985).

[21] K. Brantervik and G. A. Niklasson, Thin Solid Films 165, 67 (1988).

[22] G. A. Niklasson, Solar Energy Materials 17(3) (1988).

[23] V. N. Pustovit and G. A. Niklasson, J. Appl. Phys. 90, 1275 (2001).

[24] J. V. Mantese, W. A. Curtin and W. W. Webb, Phys. Rev. B 33, 7897 (1986).

[25] E. Tuncer, Phil. Mag. Lett 85, 269 (2005).

[26] O. Wiener, Der Abhandlungen der Mathematisch-Physischen Klasse der Königl. Sachsischen Gesellschaft der Wissenschaften 32, 509 (1912). 